\title{
EXTREMAL PROBLEMS FOR ANALYTIC FUNCTIONS WITH POSITIVE REAL PART AND APPLICATIONS
}

\author{
BY \\ M. S. ROBERTSON
}

1. Introduction. Let $\mathscr{P}$ denote the class of regular functions $P(z), P(0)=1$, with positive real part, $\operatorname{Re} P(z)>0$, in $|z|<1$, where

$$
P(z)=1+p_{1} z+p_{2} z^{2}+\cdots+p_{n} z^{n}+\cdots .
$$

Let $S$ denote the class of functions $f(z)$, regular and schlicht in $|z|<1$, normalized so that $f(0)=0, f^{\prime}(0)=1$, and where

$$
f(z)=z+a_{2} z^{2}+\cdots+a_{n} z^{n}+\cdots .
$$

Let $\Sigma$ denote the class of normalized functions $F(z)$, regular and schlicht in $0<|z|<1$, with a simple pole at the origin, and where

$$
F(z)=\frac{1}{z}+\alpha_{0}+\alpha_{1} z+\cdots+\alpha_{n} z^{n}+\cdots .
$$

Also let $\Sigma^{*}$ denote the subclass of $\Sigma$ consisting of the functions $F(z)$ which are starlike with respect to the origin in $0<|z|<1$.

There are several subclasses of $S$ and $\Sigma$ whose definition depends upon a connection between $f(z)$ and $P(z)$, or between $F(z)$ and $P(z)$. Problems associated with these various classes frequently involve the task of finding the value on $|z|=r<1$ of

$$
\underset{P \in \mathscr{P}}{\operatorname{minimum}} \operatorname{Re} F\left(P(z), z P^{\prime}(z)\right)
$$

for a given function $F(u, v)$, analytic in the plane of $v$, and in the half-plane $\operatorname{Re} u>0$. For example, if $F(z)$, given by (1.3), is a member of $\Sigma^{*}$ what is the radius $R_{k}^{*}$ of the largest circle $|z|=R_{k}^{*}$ such that every member of $\Sigma^{*}$ is convex for $0<|z| \leqq R_{k}^{*}<1$ ? We shall call $R_{k}^{*}$ the radius of convexity for the class $\Sigma^{*}$. Then, since

$$
\begin{gathered}
\frac{z F^{\prime}(z)}{F(z)}=-P(z), \\
-\left\{1+\frac{z F^{\prime \prime}(z)}{F^{\prime}(z)}\right\}=P(z)-\frac{z P^{\prime}(z)}{P(z)},
\end{gathered}
$$

it follows that $R_{k}^{*}$ is the radius of the largest circle $|z|=r$ within which

Received by the editors February 20, 1962. 


$$
\operatorname{Re}\left\{P(z)-\frac{z P^{\prime}(z)}{P(z)}\right\}>0 .
$$

The problem is therefore resolved by the solution of (1.4) for the special case where

$$
F(u, v)=u-v \cdot u^{-1} .
$$

The solution of this problem appears in the proof of Theorems 3 and 4 in $\$ 5$ of this paper where it is shown that $R_{k}^{*}=3^{-1 / 2}=0.577 \cdots$. It is interesting to note that the corresponding problem for the class $\Sigma$ has been studied and solved by Golusin in a series of papers $[2 ; 3 ; 4 ; 5 ; 6]$; see also Gelfer [1]. The radius of convexity, $R_{k}$, for the class $\Sigma$ is given by a root of the equation

$$
\frac{E(r)}{K(r)}+\frac{r^{2}}{8}-\frac{7}{8}=0, \quad r=R_{k}=0.559 \cdots,
$$

where

$$
\begin{aligned}
& E(k)=\int_{0}^{1}\left(\frac{1-k^{2} x^{2}}{1-x^{2}}\right)^{1 / 2} d x, \\
& K(k)=\int_{0}^{1}\left[\left(1-x^{2}\right)\left(1-k^{2} x^{2}\right)\right]^{-1 / 2} d x .
\end{aligned}
$$

For the class $\Sigma$, the extremal function maps the unit circle on a slit domain $D$. Since $D$ is not starlike with respect to the origin we have $R_{k}<R_{k}^{*}$. But for the class $S$ the radius of convexity is $2-3^{1 / 2}[10]$, and the extremal function $z(1-\varepsilon z)^{-2}$, $|\varepsilon|=1$, is starlike in $|z|<1$.

Another example can be seen in the case where $F(z)$ of (1.3) omits the value zero and is starlike in the direction of the real axis for $|z|<1$. This means that the real axis cuts the map of $|z|=r<1$ by $w=F(z)$ in exactly two points for every $r$ near 1 . Then [11]

$$
\{F(z)\}^{-1}=h_{v}\left(e^{-i \mu} z\right)(\cos \mu+i \sin \mu P(z))
$$

where $P(z) \in \mathscr{P}, \sin \mu \geqq 0$ and

$$
h_{\nu}(z)=z\left(1-2 z \cos v+z^{2}\right)^{-1} .
$$

Although $F(z)$ need not be schlicht in the unit circle, we may ask what is the largest value of $R$ such that every such $F(z)$ is schlicht and starlike with respect to the origin in $0<|z| \leqq R$.

Since

$$
\begin{aligned}
\operatorname{Re}\left\{\frac{-z F^{\prime}(z)}{F(z)}\right\} & =\operatorname{Re}\left\{\frac{1-e^{-2 i \mu} z^{2}}{1-2 e^{-i \mu} z \cos v+e^{-2 i \mu} z^{2}}\right\}+\operatorname{Re}\left\{\frac{i \sin \mu z P^{\prime}(z)}{\cos \mu+i \sin \mu P(z)}\right\} \\
& \geqq \frac{1-r}{1+r}-\frac{2 r}{1-r^{2}} \geqq 0, \quad r \geqq 2-3^{1 / 2},
\end{aligned}
$$


it follows that $R \geqq 2-3^{1 / 2}$. In (1.14) we have used the inequality

$$
\operatorname{Re}\left\{\frac{i \sin \mu z P^{\prime}(z)}{\cos \mu+i \sin \mu P(z)}\right\} \geqq \frac{-2 r}{1-r^{2}}, \quad r<1,
$$

which will be established in $\S 6$ of this paper. We may look upon (1.15) as a particular case of the more general problem (1.4) where here $F(u, v)$ has the special form

$$
F(u, v)=v \cdot(u-i \cot \mu)^{-1} .
$$

Equality is attained in (1.14) for $z=-i r, v=0, \mu=\pi / 2, P(z)=(1-i z)(1+i z)^{-1}$, $F(z)=(1+i z)^{3} \cdot\left(z-i z^{2}\right)^{-1}$. The value $R=2-3^{1 / 2}$ is best possible since $F^{\prime}(z)$, vanishes on $|z|=2-3^{1 / 2}$.

Several other examples involving functions $F(u, v)$ might be cited. But we turn now to the general problem indicated in (1.4). Since $P(z)$ may be represented by the Herglotz Stieltjes integral formula

$$
P(z)=\int_{0}^{2 \pi} \frac{1+z e^{i \theta}}{1-z e^{i \theta}} d \alpha(\theta),
$$

where $\alpha(\theta)$ is a nondecreasing function in $[0,2 \pi]$, normalized so that

$$
\int_{0}^{2 \pi} d \alpha(\theta)=1
$$

we may approximate $P(z)$ by rational functions of the form

$$
P_{n}(z)=\sum_{k=1}^{n} p_{k} \frac{1+\varepsilon_{k} z}{1-\varepsilon_{k} z},\left|\varepsilon_{k}\right|=1,0 \leqq p_{k} \leqq 1, \sum_{1}^{n} p_{k}=1 .
$$

We shall show that the extremal functions for (1.4) are always of the form (1.19) with $n \leqq 2$ for all functions $F(u, v)$ of the class considered. The specific values of the parameters $p_{k}, \varepsilon_{k}$ will depend upon the given $F(u, v)$. Their values are sometimes difficult to compute but will be obtained for the examples (1.8) and (1.16). The main theorem of this paper is the following:

THEOREM 1. If $F(u, v)$ is analytic in the v-plane and in the half-plane $\operatorname{Re} u>0$, and if $P(z) \in \mathscr{P}$, then on $|z|=r<1$

$$
\min _{p \in \mathscr{F}} \operatorname{Re} F\left\{P(z), z P^{\prime}(z)\right\}=\min _{\alpha, \theta, \phi} \operatorname{Re} F\left\{P_{0}(z), z P_{0}^{\prime}(z)\right\}
$$

where

$$
\begin{gathered}
P_{0}(z)=\frac{1+\alpha}{2}\left(\frac{1+z e^{i \theta}}{1-z e^{i \theta}}\right)+\frac{1-\alpha}{2}\left(\frac{1+z e^{-i \theta}}{1-z e^{-i \theta}}\right), z=r e^{i \phi}, \\
-1 \leqq \alpha \leqq 1, \quad 0 \leqq \theta \leqq 2 \pi, \quad 0 \leqq \phi \leqq 2 \pi .
\end{gathered}
$$


A special case of Theorem 1, which we give as Theorem 2, is of some interest too. The proof follows by the method of variations used in the proof of Theorem 1 , but an independent proof by the method of subordination suffices and is simpler.

THEOREM 2. If $F(u)$ is analytic in the half-plane $\operatorname{Re} u>0$ and if $P(z) \in \mathscr{P}$, then on $|z|=r<1$

$$
\min _{p \in \mathscr{P}} \operatorname{Re} F(P(z))=\min _{|z|=r} \operatorname{Re} F\left(\frac{1+z}{1-z}\right) .
$$

Using Theorem 1 we find for the special case (1.7) the following result given as Theorem 3.

THEOREM 3. Let $P(z) \in \mathscr{P}$. Then

$$
\operatorname{Re}\left\{P(z)-\frac{z P^{\prime}(z)}{P(z)}\right\} \geqq 0 \quad \text { for } \quad|z| \leqq 3^{-1 / 2}
$$

Equality in (1.23) is attained on $|z|=3^{-1 / 2}$ only for the function

$$
P_{0}(z)=\frac{1+3^{-1 / 2}}{2}\left(\frac{1+\varepsilon z}{1-\varepsilon z}\right)+\frac{1-3^{-1 / 2}}{2}\left(\frac{1-\varepsilon z}{1+\varepsilon z}\right), \quad|\varepsilon|=1 \text {. }
$$

Because of (1.6) Theorem 3 implies the following Theorem 4.

THEOREM 4. Let $F(z) \in \Sigma^{*}$, where

$$
F(z)=\frac{1}{z}+\alpha_{0}+\alpha_{1} z+\cdots+\alpha_{n} z^{n}+\cdots, \quad 0<|z|<1 .
$$

Then $F(z)$ is convex in $|z| \leqq R$ where $R>3^{-1 / 2}$ except for the function

$$
F_{0}(z)=z^{-1}(1+\varepsilon z)^{1-3^{-1 / 2}} \cdot(1-\varepsilon z)^{1+3^{-1 / 2}},|\varepsilon|=1 \text {, }
$$

in which case $R=3^{-1 / 2}=0.577 \cdots$.

Applying Theorem 1 to the special case (1.16) we have the theorem

THEOREM 5. Let

$$
F(z)=\frac{1}{z}+\alpha_{0}+\alpha_{1} z+\cdots+\alpha_{n} z^{n}+\cdots
$$

be regular and starlike in the direction of the real axis (but not necessarily schlicht) and omit the value 0 for $0<|z|<1$. Then $F(z)$ is schlicht and starlike with respect to the origin in $0<|z|<2-3^{1 / 2}$. The function $F(z)=(1+i z)^{3}$ $\cdot\left(z-i z^{2}\right)^{-1}$ shows that $2-3^{1 / 2}$ cannot be replaced by a larger number.

The proof of Theorem 1 depends upon an application of the variational formula recently obtained by the author [12] for functions of class $\mathscr{P}$ :

$$
P^{*}(z)=P(z)-\rho^{2}\left(1-\left|z_{0}\right|^{2}\right) \lambda\left(z, z_{0}\right)+o\left(\rho^{2}\right),
$$


where

$$
\begin{aligned}
\lambda\left(z, z_{0}\right)= & \left(\frac{z_{0} P^{\prime}(z)}{P\left(z_{0}\right)}-1\right) \frac{z e^{i \theta}}{z_{0}\left(z_{0}-z\right)}+\left(\frac{z_{0} P(z)}{P\left(z_{0}\right)}-z\right) \frac{z e^{i \theta}}{z_{0}\left(z_{0}-z\right)^{2}} \\
& +\frac{P^{\prime}(z)}{\left(P\left(z_{0}\right)\right)^{-}}\left(\frac{z^{2} e^{-i \theta}}{1-\bar{z}_{0} z}\right)+\left(\frac{P(z)}{\left(P\left(z_{0}\right)\right)^{-}}+1\right) \frac{z e^{-i \theta}}{\left(1-\bar{z}_{0} z\right)^{2}}
\end{aligned}
$$

$\left|z_{0}\right|<1, \theta$ real and arbitrary, $\rho$ real and small. Here and throughout the paper ( )indicates the complex conjugate. Formula (1.28) was derived [12] from an equivalent formula for a starlike univalent function $f(z)$ regular in $|z|<1$ due to Hummel [7].

2. Proof of Theorem 1. From (1.28) we have

$$
z P^{*^{\prime}}(z)=z P^{\prime}(z)-\rho^{2}\left(1-\left|z_{0}\right|^{2}\right) z \lambda^{\prime}\left(z, z_{0}\right)+o\left(\rho^{2}\right)
$$

where ()$^{\prime}$ denotes differentiation with respect to $z$. From (1.29) we have

where

$$
\lambda^{\prime}\left(z, z_{0}\right)=\mu\left(z, z_{0}\right) e^{i \theta}+v\left(z, z_{0}\right) e^{-i \theta}
$$

$$
\begin{aligned}
\mu\left(z, z_{0}\right)= & \frac{P^{\prime \prime}(z)}{P\left(z_{0}\right)} \frac{z}{z_{0}-z}+\left(\frac{z_{0} P^{\prime}(z)}{P\left(z_{0}\right)}-1\right) \frac{\left(z+z_{0}\right)}{z_{0}\left(z_{0}-z\right)^{2}} \\
& +\left(\frac{z_{0} P(z)}{P\left(z_{0}\right)}-z\right) \frac{\left(z_{0}+z\right)}{z_{0}\left(z_{0}-z\right)^{3}}, \\
v\left(z, z_{0}\right)= & \frac{P^{\prime \prime}(z)}{\left(P\left(z_{0}\right)\right)^{-}}\left(\frac{z^{2}}{1-\bar{z}_{0} z}\right)+\frac{P^{\prime}(z)}{\left(P\left(z_{0}\right)\right)^{-}} \cdot \frac{\left(3 z-\bar{z}_{0} z^{2}\right)}{\left(1-\bar{z}_{0} z\right)^{2}} \\
& +\left(\frac{P(z)}{\left(P\left(z_{0}\right)\right)^{-}}+1\right) \frac{\left(1+\bar{z}_{0} z\right)}{\left(1-\bar{z}_{0} z\right)^{3}} .
\end{aligned}
$$

Let $F(u, v)$ satisfy the conditions of Theorem 1. We obviously may assume $F(u, v)$ not a constant function since Theorem 1 is trivially true otherwise. In (1.4) we may take $z=r>0$. For if $P_{0}(z)$ is an extremal function (the family $\mathscr{P}$ is compact) for

$$
\min _{P \in \mathscr{P}} \operatorname{Re} F\left(P(r), r P^{\prime}(r)\right)=\operatorname{Re} F\left(P_{0}(r), r P_{0}^{\prime}(r)\right)
$$

then since $P\left(e^{i \theta} z\right) \in \mathscr{P}$, we have for any $P(z)$

$$
\operatorname{Re} F\left(P\left(r e^{i \theta}\right), r e^{i \theta} P^{\prime}\left(r e^{i \theta}\right)\right) \geqq \operatorname{Re} F\left(P_{0}(r), r P_{0}^{\prime}(r)\right)
$$

by (2.5) where $P\left(e^{i \theta} z\right)$ replaces $P(z)$, and $z=r$. (2.6) may be written as

$$
\operatorname{Re} F\left(P(z), z P^{\prime}(z)\right) \geqq \operatorname{Re} F\left(P_{0}(r), r P_{0}^{\prime}(r)\right), \quad z=r e^{i \theta} .
$$

From (2.7) it follows that on $|z|=r$

$$
\min _{P \in \mathscr{G}} \operatorname{Re} F\left(P(z), z P^{\prime}(z)\right)=\operatorname{Re} F\left(P_{0}(r), r P_{0}^{\prime}(r)\right)
$$


Consequently, in obtaining the value of the expression (1.4) we assume $z=r>0$.

We shall need the following lemma.

LEMMA 1. $\left[P^{\prime}(r) F_{u}\left(P(r), r P^{\prime}(r)\right)+F_{v}\left(P(r), r P^{\prime}(r)\right)\left\{r P^{\prime \prime}(r)+P^{\prime}(r)\right\}\right]$ is a real number for an extremal function $P(z)=P_{0}(z)$ in (2.8).

Proof. Since $P_{0}\left(z e^{i \theta}\right) \in \mathscr{P}$ for all $\theta$ we have from (2.8)

$$
\begin{aligned}
\operatorname{Re} F\left(P_{0}\left(r e^{i \theta}\right), r e^{i \theta} P_{0}^{\prime}\left(r e^{i \theta}\right)\right) & \geqq \operatorname{Re} F\left(P_{0}(r), r P_{0}^{\prime}(r)\right), \\
\min _{|z|=r} \operatorname{Re} F\left(P_{0}^{\prime}(z), z P_{0}(z)\right) & =\operatorname{Re} F\left(P_{0}(r), r P_{0}^{\prime}(r)\right) .
\end{aligned}
$$

For $z=r e^{i \theta}$ and a minimum in (2.10) at $\theta=0$ we have

$$
\left.\frac{\partial}{\partial \theta} \operatorname{Re} F\left(P_{0}(z), z P_{0}^{\prime}(z)\right)\right|_{\theta=0}=0 \text {. }
$$

Since $F\left(P_{0}, z P_{0}^{\prime}\right)$ is an analytic function of $z$, the Cauchy-Riemann equations give

$$
\begin{array}{r}
I_{m} \frac{\partial}{\partial r} F\left(P_{0}(r), r P_{0}^{\prime}(r)\right)=-\left.\frac{1}{r} \frac{\partial}{\partial \theta} \operatorname{Re} F\left(P_{0}(z), z P_{0}^{\prime}(z)\right)\right|_{\theta=0}=0 \\
I_{m}\left[P_{0}^{\prime}(r) F_{u}\left(P_{0}(r), r P_{0}^{\prime}(r)\right)+F_{v}\left(P_{0}(r), r P_{0}^{\prime}(r)\right)\left\{r P_{0}^{\prime \prime}(r)+P_{0}^{\prime}(r)\right\}\right]=0 .
\end{array}
$$

The Taylor series for $F(u, v)$ gives

$$
F(a+h, b+k)=F(a, b)+F_{u}(a, b) h+F_{v}(a, b) k+\cdots .
$$

Take

$$
\begin{aligned}
& a=P(r), b=r P^{\prime}(r), h=-\rho^{2}\left(1-\left|z_{0}\right|^{2}\right) \lambda\left(r, z_{0}\right)+o\left(\rho^{2}\right), \\
& k=-\rho^{2}\left(1-\left|z_{0}\right|^{2}\right) r \lambda^{\prime}\left(r, z_{0}\right)+o\left(\rho^{2}\right) .
\end{aligned}
$$

Then

$$
\begin{aligned}
& F\left(P^{*}(r), r P^{* \prime}(r)\right)-F\left(P(r), r P^{\prime}(r)\right) \\
& \quad=\left[F_{u}\left(P, r P^{\prime}\right) \lambda\left(r, z_{0}\right)+r \lambda^{\prime}\left(r, z_{0}\right) F_{v}\left(P, r P^{\prime}\right)\right]\left(1-\left|z_{0}\right|^{2}\right) \rho^{2}+o\left(\rho^{2}\right)
\end{aligned}
$$

If $P(r)=P_{0}(r)$, an extremal function, then since

$$
\operatorname{Re} F\left(P^{*}, r P^{* \prime}\right) \geqq \operatorname{Re} F\left(P_{0}, r P_{0}^{\prime}\right)
$$

we have from (2.15)

$$
\operatorname{Re}\left[F_{u}\left(P_{0}, r P_{0}^{\prime}\right) \lambda\left(r, z_{0}\right)+r \lambda^{\prime}\left(r, z_{0}\right) F_{v}\left(P_{0}, r P_{0}^{\prime}(r)\right)\right] \leqq 0
$$

for $r$ fixed, and all $\theta$.

Since $\operatorname{Re}\left(e^{-i \theta} w\right)=\operatorname{Re}\left(e^{i \theta} \bar{w}\right)$ we have for all $\theta$ that

$$
\operatorname{Re}\left(e^{i \theta} B\right) \leqq 0
$$

where $B$ is the expression defined as 


$$
\begin{aligned}
& B=\left[\left(\frac{z_{0}}{P_{0}\left(z_{0}\right)} P_{0}^{\prime}(r)-1\right) \frac{r}{z_{0}\left(z_{0}-r\right)}+\left(\frac{z_{0} P_{0}(r)}{P_{0}\left(z_{0}\right)}-r\right) \frac{r}{z_{0}\left(z_{0}-r\right)^{2}}\right] F_{u} \\
& +\left[\frac{\left(P_{0}^{\prime}(r)\right)^{-}}{P_{0}\left(z_{0}\right)} \cdot \frac{r^{2}}{1-r z_{0}}+\left(\frac{\left(P_{0}(r)\right)^{-}}{P_{0}\left(z_{0}\right)}+1\right) \frac{r}{\left(1-r z_{0}\right)^{2}}\right]\left(F_{u}\right)^{-} \\
& +\left[\frac{P_{0}^{\prime \prime}(r)}{P_{0}\left(z_{0}\right)} \cdot \frac{r}{z_{0}-r}+\left(\frac{z_{0} P_{0}^{\prime}(r)}{P_{0}\left(z_{0}\right)}-1\right) \frac{\left(z_{0}+r\right)}{z_{0}\left(z_{0}-r\right)^{2}}+\left(\frac{z_{0} P_{0}(r)}{P_{0}\left(z_{0}\right)}-r\right) \frac{\left(z_{0}+r\right)}{z_{0}\left(z_{0}-r\right)^{3}}\right] r F_{v} \\
& +\left[\frac{\left(P_{0}^{\prime \prime}(r)\right)^{-}}{P_{0}\left(z_{0}\right)} \cdot \frac{r^{2}}{\left(1-r z_{0}\right)}+\frac{\left(P_{0}^{\prime}(r)\right)^{-}}{P_{0}\left(z_{0}\right)} \frac{\left(3 r-r^{2} z_{0}\right)}{\left(1-r z_{0}\right)^{2}}+\left(\frac{\left(P_{0}(r)\right)^{-}}{P_{0}\left(z_{0}\right)}+1\right) \frac{\left(1+r z_{0}\right)}{\left(1-r z_{0}\right)^{3}}\right] r\left(F_{v}\right)^{-} .
\end{aligned}
$$

Solving the equation $B=0$ (which follows from (2.17) for $P_{0}\left(z_{0}\right)$ and replacing $z_{0}$ by $z$ we obtain the extremal function to be a rational function of the form

$$
P_{0}(z)=\sum_{0}^{5} \alpha_{k} z^{k} \div \sum_{0}^{4} \beta_{k} z^{k}
$$

where

$$
\begin{aligned}
& \sum_{0}^{5} \alpha_{k} z^{k}=(1-r z)^{3}\left[(z-r)^{2}\left(P_{0}^{\prime} F_{u}+r P_{0}^{\prime \prime} F_{v}\right)+(z-r) P_{0} F_{u}+\left(z^{2}-r^{2}\right) P_{0}^{\prime} F_{v}\right. \\
& \left.+(z+r) P_{0} F_{v}\right] \\
& +(z-r)^{3}\left[\begin{array}{l}
r(1-r z)^{2}\left(P_{0}^{\prime} F_{u}+r P_{0}^{\prime \prime} F_{v}\right)^{-}+(1-r z)\left\{\left(P_{0} F_{u}\right)^{-}+\left(3 r-r^{2} z\right)\left(P_{0}^{\prime} F_{v}\right)^{-}\right\} \\
+(1+r z)\left(P_{0} F_{v}\right)^{-}
\end{array}\right]
\end{aligned}
$$

where the constants $P_{0}, P_{0}^{\prime}, P_{0}^{\prime \prime}, F_{u}=F_{u}\left(r P_{0}(r), r P_{0}^{\prime}(r)\right)$, and $F_{v}$ are evaluated at the fixed point $r$. Similarly,

$$
\begin{aligned}
& \sum_{0}^{4} \beta_{k} z^{k}=(z-r)(1-r z)\left\{(1-r z)^{2} F_{u}-(z-r)^{2}\left(F_{u}\right)^{-}\right\} \\
&+(z+r)(1-r z)^{3} F_{v}-(1+r z)(z-r)^{3}\left(F_{v}\right)^{-}
\end{aligned}
$$

We should notice in passing that if $F(u, v)$ is independent of $v$, then $F_{v}$ vanishes identically in (2.20) and (2.21). In this case $(z-r)(1-r z)$ is a factor of the numerator and denominator of the extremal function $P_{0}(z)$ in (2.19). Thus the degree of each polynomial is decreased by 2 .

From (2.20) we find that $\alpha_{5}$ has the value

$$
\alpha_{5}=-2 i r^{3} I_{m}\left[P_{0}^{\prime} F_{u}+\left(P_{0}^{\prime}+r P_{0}^{\prime \prime}\right) F_{v}\right]=0
$$

by Lemma 1 . Since $\alpha_{5}=0$ the extremal function $P_{0}(z)$ minimizing $\operatorname{Re} F\left(P(r), r P^{\prime}(r)\right)$ over the class $\mathscr{P}$ for a fixed $r, 0<r<1$, is of the form

$$
P_{0}(z)=\frac{\alpha_{0}+\alpha_{1} z+\alpha_{2} z^{2}+\alpha_{3} z^{3}+\alpha_{4} z^{4}}{\beta_{0}+\beta_{1} z+\beta_{2} z^{2}+\beta_{3} z^{3}+\beta_{4} z^{4}} .
$$

It is remarkable that the degree of $P_{0}(z)$ is quite independent of the function $F(u, v)$ chosen and results solely from the form of the variational formula (1.28) 
and the fact that $F(u, v)$ is a function involving no higher derivatives of $P(z)$ than the first. We shall see presently, however, that the degree of the polynomials in the numerator and denominator of $P_{0}(z)$ may be reduced to degree 2 .

From (2.20) we calculate the coefficients $\alpha_{k}$ and simplify the expressions by means of Lemma 1 , or more explicitly by equation (2.13).

$$
\begin{aligned}
\alpha_{0}= & r^{2}\left(P_{0}^{\prime} F_{u}+P_{0}^{\prime} F_{v}+r P_{0}^{\prime \prime} F_{v}\right)-r P_{0}\left(F_{u}-F_{v}\right)-2 r^{2} P_{0}^{\prime} F_{v} \\
& -r^{4}\left(P_{0}^{\prime} F_{u}+P_{0}^{\prime} F_{v}+r P_{0}^{\prime \prime} F_{v}\right)^{-}-r^{3}\left(P_{0} F_{u}+P_{0} F_{v}\right)^{-}-2 r^{4}\left(P_{0}^{\prime} F_{v}\right)^{-} \\
= & \left(r^{2}-r^{4}\right)\left(P_{0}^{\prime} F_{u}+P_{0}^{\prime} F_{v}+r P_{0}^{\prime \prime} F_{v}\right)-r P_{0}\left(F_{u}-F_{v}\right)-r^{3}\left(P_{0} F_{u}+P_{0} F_{v}\right)^{-} \\
& -2 r^{2} P_{0}^{\prime} F_{v}-2 r^{4}\left(P_{0}^{\prime} F_{v}\right)^{-} .
\end{aligned}
$$

(2.25) $\alpha_{1}=-\left(P_{0}^{\prime} F_{u}+P_{0}^{\prime} F_{v}+r P_{0}^{\prime \prime} F_{v}\right)\left(3 r^{3}+2 r\right)+P_{0} F_{u}\left(3 r^{2}+1\right)+\left(P_{0} F_{u}\right)-\cdot\left(3 r^{2}+r^{4}\right)$

$$
\begin{aligned}
& -\left(P_{0}^{\prime} F_{u}+P_{0}^{\prime} F_{v}+r P_{0}^{\prime \prime} F_{v}\right)^{-} \cdot\left(3 r^{3}+2 r^{5}\right)+\left(6 r^{3}+2 r\right) P_{0}^{\prime} F_{v} \\
& +\left(6 r^{3}+2 r^{5}\right)\left(P_{0}^{\prime} F_{v}\right)^{-}+\left(1-3 r^{2}\right) P_{0} F_{v}+\left(3 r^{2}-r^{4}\right)\left(P_{0} F_{v}\right)^{-} \\
= & -\left(P_{0}^{\prime} F_{u}+P_{0}^{\prime} F_{v}+r P_{0}^{\prime \prime} F_{v}\right)\left(2 r-2 r^{5}\right)+P_{0} F_{u}\left(3 r^{2}+1\right) \\
& +\left(P_{0} F_{u}\right)^{-} \cdot\left(3 r^{2}+r^{4}\right)+\left(6 r^{3}+2 r\right) P_{0}^{\prime} F_{v}+\left(6 r^{3}+2 r^{5}\right)\left(P_{0}^{\prime} F_{v}\right)^{-} \\
& +\left(1-3 r^{2}\right) P_{0} F_{v}+\left(3 r^{2}-r^{4}\right)\left(P_{0} F_{v}\right)^{-} .
\end{aligned}
$$

(2.26) $\alpha_{2}=\left(P_{0}^{\prime} F_{u}+P_{0}^{\prime} F_{v}+r P_{0}^{\prime \prime} F_{v}\right)\left(1+6 r^{2}+3 r^{4}\right)-\left\{P_{0} F_{u}+\left(P_{0} F_{u}\right)^{-}\right\}\left(3 r^{3}+3 r\right)$

$$
\begin{aligned}
& -\left\{P_{0}^{\prime} F_{v}+\left(P_{0}^{\prime} F_{v}\right)^{-}\right\}\left(6 r^{2}+6 r^{4}\right)+\left\{P_{0} F_{v}+\left(P_{0} F_{v}\right)^{-}\right\} \\
& -\left(P_{0}^{\prime} F_{u}+P_{0}^{\prime} F_{v}+r P_{0}^{\prime \prime} F_{v}\right)^{-} \cdot\left(r^{6}+6 r^{4}+3 r^{2}\right) \\
= & \left(1-r^{2}\right)\left(1+4 r^{2}+r^{4}\right)\left(P_{0}^{\prime} F_{u}+P_{0}^{\prime} F_{v}+r P_{0}^{\prime \prime} F_{v}\right)-\left(6 r+6 r^{3}\right) \operatorname{Re}\left(P_{0} F_{u}\right) \\
& -\left(6 r-6 r^{3}\right) \operatorname{Re}\left(P_{0} F_{v}\right)-\left(6 r^{2}+6 r^{4}\right) \operatorname{Re}\left(P_{0}^{\prime} F_{v}\right)
\end{aligned}
$$

$=\mathbf{a}$ real number.

$$
\begin{aligned}
\alpha_{3}= & -\left(P_{0}^{\prime} F_{u}+r P_{0}^{\prime \prime} F_{v}\right)\left(6 r^{3}+r^{5}+3 r\right)+\left(r^{4}+3 r^{2}\right) P_{0} F_{u}+\left(r^{5}-3 r\right) P_{0}^{\prime} F_{v} \\
& +\left(3 r^{2}-r^{4}\right) P_{0} F_{v}+\left(r+6 r^{3}+3 r^{5}\right)\left(P_{0}^{\prime} F_{u}+r P_{0}^{\prime \prime} F_{v}\right)^{-} \\
& +\left(3 r^{2}+1\right)\left(P_{0} F_{u}\right)^{-}+\left(3 r^{5}+12 r^{3}+3 r\right)\left(P_{0}^{\prime} F_{v}\right)^{-}+\left(1-3 r^{2}\right)\left(P_{0} F_{v}\right)^{-} \\
= & -\left(P_{0}^{\prime} F_{u}+P_{0}^{\prime} F_{v}+r P_{0}^{\prime \prime} F_{v}\right)\left(2 r-2 r^{5}\right)+P_{0}^{\prime} F_{v}\left(2 r^{5}+6 r^{3}\right) \\
& +\left(P_{0}^{\prime} F_{v}\right)^{-} \cdot\left(6 r^{3}+2 r\right)+P_{0} F_{u}\left(r^{4}+3 r^{2}\right)+\left(P_{0} F_{u}\right)^{-} \cdot\left(3 r^{2}+1\right) \\
& +P_{0} F_{v}\left(3 r^{2}-r^{4}\right)+\left(P_{0} F_{v}\right)^{-} \cdot\left(1-3 r^{2}\right) \\
= & \bar{\alpha}_{1} .
\end{aligned}
$$




$$
\begin{aligned}
\alpha_{4}= & \left(P_{0}^{\prime} F_{u}+r P_{0}^{\prime \prime} F_{v}\right)\left(3 r^{2}+2 r^{4}\right)-r^{3} P_{0} F_{u}+3 r^{2} P_{0}^{\bar{\gamma}} F_{v}-r^{3} P_{0} F_{v} \\
& -\left(P_{0}^{\prime} F_{u}+r P_{0}^{\prime \prime} F_{v}\right)^{-} \cdot\left(3 r^{4}+2 r^{2}\right)-r\left(P_{0} F_{u}\right)^{-}-\left(3 r^{4}+4 r^{2}\right)\left(P_{0}^{\prime} F_{v}\right)^{-} \\
& +r\left(P_{0} F_{v}\right)^{-} \\
= & \left(r^{2}-r^{4}\right)\left(P_{0}^{\prime} F_{u}+P_{0}^{\prime} F_{v}+r P_{0}^{\prime \prime} F_{v}\right)-r\left(P_{0} F_{u}-P_{0} F_{v}\right)^{-} \\
& -r^{3}\left(P_{0} F_{u}+P_{0} F_{v}\right)-2 r^{2}\left(P_{0}^{\prime} F_{v}\right)^{-}-2 r^{4}\left(P_{0}^{\prime} F_{v}\right) \\
= & \bar{\alpha}_{0} .
\end{aligned}
$$

From (2.21) we also calculate the coefficients $\beta_{k}$ and obtain

(2.29) $\beta_{0}=r^{3}\left(F_{u}\right)^{-}-r F_{u}+r^{3}\left(F_{v}\right)^{-}+r F_{v}$,

(2.30) $\beta_{1}=-\left(r^{4}+3 r^{2}\right)\left(F_{u}\right)^{-}+\left(3 r^{2}+1\right) F_{u}-\left(3 r^{2}-r^{4}\right)\left(F_{v}\right)^{-}+\left(1-3 r^{2}\right) F_{v}$,

(2.31) $\beta_{2}=-\left(3 r^{3}+3 r\right)\left\{F_{u}-\left(F_{u}\right)^{-}\right\}-\left(3 r-3 r^{3}\right)\left\{F_{v}-\left(F_{v}\right)^{-}\right\}$

$=$ a pure imaginary number,

(2.32) $\beta_{3}=-\bar{\beta}_{1}$,

(2.33) $\beta_{4}=-\bar{\beta}_{0}$.

Since $\alpha_{4}=\bar{\alpha}_{0}, \alpha_{3}=\bar{\alpha}_{1}, \alpha_{2}$ real, $\beta_{4}=-\bar{\beta}_{0}, \beta_{3}=-\bar{\beta}_{1}, \beta_{2}$ pure imaginary, $P_{0}(z)$ in (2.23) may now be written as

$$
P_{0}(z)=\frac{\alpha_{0} z^{-2}+\alpha_{1} z^{-1}+\alpha_{2}+\bar{\alpha}_{1} z+\bar{\alpha}_{0} z^{2}}{\beta_{0} z^{-2}+\beta_{1} z^{-1}+\beta_{2}-\bar{\beta}_{1} z-\bar{\beta}_{0} z^{2}}=\frac{Q(z)}{R(z)} .
$$

Since $P(0)=1$, we also have $\alpha_{0}=\beta_{0}$. It is seen from (2.34) that we have identically

$$
P_{0}(z)+\left(P_{0}\left(\frac{1}{\bar{z}}\right)\right)^{-}=0
$$

Thus the real part of $P_{0}(z)$ vanishes on $|z|=1$. Indeed, $Q\left(e^{i \theta}\right)$ is real and $R\left(e^{i \theta}\right)$ is purely imaginary. As we shall soon see $Q(z)$ and $R(z)$ have zeros in common. Let $m$ denote the number of poles of $P_{0}(z)$, that is the number of zeros of $R(z)$ distinct from the zeros of $Q(z)$. Because of (2.35) and the fact that $\operatorname{Re} P_{0}(z)>0$ in $|z|<1$ with $P_{0}(0)=1$, we may write $P_{0}(z)$ of $(2.34)$ in the form

$$
P_{0}(z)=\sum_{k=1}^{m} p_{k}\left(\frac{1+\varepsilon_{k} z}{1-\varepsilon_{k} z}\right)
$$

where $0<p_{k}<1, \sum_{1}^{m} p_{k}=1,\left|\varepsilon_{k}\right|=1$ and $m \leqq 4$ (compare formula (3.16) of [12]).

In $\$ 3$ we shall show that $Q\left(e^{i \theta}\right)$, which is real, has the same sign near each of any two consecutive simple poles $\bar{\varepsilon}_{k}, \bar{\varepsilon}_{k+1}$ in the interval between them on $|z|=1$. 
Thus $Q\left(e^{\prime 0}\right)$ has an even number of zeros between each of the poles. It will also be shown that $Q\left(e^{i \theta}\right)$ has at least one zero between consecutive poles. It then follows that $Q(z)$ has at least $2 m$ zeros on $|z|=1$ but certainly not more than 4 zeros. Thus $m \leqq 2$. When this fact has been established $P_{0}(z)$ in $(2.36)$ may be written with $z=r>0$ in the form

$$
P_{0}(r)=\frac{1+\alpha}{2}\left(\frac{1+r \varepsilon_{1}}{1-r \varepsilon_{1}}\right)+\frac{1-\alpha}{2}\left(\frac{1+r \varepsilon_{2}}{1-r \varepsilon_{2}}\right)
$$

where $-1 \leqq \alpha \leqq 1,\left|\varepsilon_{1}\right|=\left|\varepsilon_{2}\right|=1$.

As in $:$. ted by (2.8) the solution of finding the value given in (1.4) for a given function $F(u, v)$ becomes one of finding the precise values of the parameters $\alpha, \varepsilon_{1}$ and $\varepsilon_{2}$ which may possibly depend upon the fixed number $r$. If we let $z=r e^{i \phi}, \varepsilon_{1}=\exp i(\phi+\theta), \varepsilon_{2}=\exp i(\phi-\theta)$ in (2.37) then (1.20) and (1.21) of Theorem 1 can replace (2.8) and (2.37), respectively.

3. The Julia variation applied to $P(z)$. Let $f(z), f(0)=0, f^{\prime}(0)=1$, map $|z|<1$ onto a domain $D$ with boundary $C$. Let $C$ be mapped into $C^{\Delta}$ by the mapping $w^{\Delta}=w+\rho \delta w$ where $\rho$ is a small real parameter and where $\rho \delta w$ is a shift of each point $w$ of $C$ in a direction normal to $C$. Let $\psi(z), \psi(0)=0$, map $|z|<1$ onto the domain $D^{\Delta}$ bounded by $C^{\Delta}$. The Julia variation formula for the function $\psi(z)$ is defined as [9]

$$
\psi(z)=f(z)+\rho \oint_{C} z f^{\prime}(z) \frac{\zeta+z}{\zeta-z} \frac{\delta w d w}{2 \pi i \zeta^{2} f^{\prime}(\zeta)^{2}}+o(\rho) .
$$

Dividing by $\psi^{\prime}(0)$ to obtain normalization we have

$$
f^{\Delta}(z)=f(z)+\rho \oint_{C}\left[z f^{\prime}(z) \frac{\zeta+z}{\zeta-z}-f(z)\right]\left[\frac{\delta w d w}{2 \pi i \zeta^{2} f^{\prime}(\zeta)^{2}}\right]+o(\rho) .
$$

Let $f(z)$ be the starlike function defined by the equations

$$
z f^{\prime}(z) \div f(z)=P_{0}(z), \quad f(0)=0, \quad f^{\prime}(0)=1,
$$

where $P_{0}(z)$ is the extremal function $Q(z) / R(z)$ of (2.34). Denote by $\phi$ the expression

$$
\phi=\frac{\delta w}{2 \pi i \zeta^{2} f^{\prime}(\zeta)^{2}}, w=f(\zeta)
$$

Then differentiation in (3.2) yields

$$
z f^{\Delta^{\prime}}(z)=z f^{\prime}(z)+\rho \oint_{C}\left[z^{2} f^{\prime \prime}(z) \frac{\zeta+z}{\zeta-z}+z f^{\prime}(z) \frac{\left(4 z \zeta-z^{2}\right)}{(\zeta-z)^{2}}\right] \phi d w+o(\rho)
$$

On dividing (3.2) by (3.5) and letting $P_{0}^{\Delta}(z)=z f^{\Delta^{\prime}}(z) \div f^{\Delta}(z)$ we have

$$
P_{0}^{\Delta}(z)=P_{0}(z)+\rho \oint_{C}\left[z P_{0}^{\prime}(z) \frac{\zeta+z}{\zeta-z}+\frac{2 \zeta z P_{0}(z)}{(\zeta-z)^{2}}\right] \phi d w+o(\rho),
$$




$$
\begin{gathered}
z P_{0}^{\Delta^{\prime}}(z)=z P_{0}^{\prime}(z)+\rho \oint_{C}\left[z^{2}\left(\frac{\zeta+z}{\zeta-z}\right) P_{0}^{\prime \prime}(z)+\frac{\left(\zeta^{2}+4 \zeta z-z^{2}\right)}{(\zeta-z)^{2}} z P_{0}^{\prime}(z)\right. \\
\left.+\frac{2 \zeta z(\zeta+z) P_{0}(z)}{(\zeta-z)^{3}}\right] \phi d w+o(\rho) \\
F\left(P_{0}^{\Delta}(z), z P_{0}^{\Delta^{\prime}}(z)\right)=F\left(P_{0}(z), z P_{0}^{\prime}(z)\right)+\rho D+o(\rho)
\end{gathered}
$$

where

where $M$ denotes

$$
D=\oint_{C} M \phi d w
$$

$$
\begin{array}{r}
M=\left[\frac{\zeta+z}{\zeta-z}\left(z^{2} P_{0}^{\prime \prime} F_{v}+z P_{0}^{\prime} F_{u}\right)+\frac{2 z \zeta F_{u} P_{0}+\left(\zeta^{2}+4 \zeta z-z^{2}\right) z P_{0}^{\prime} F_{v}}{(\zeta-z)^{2}}\right. \\
\left.+\frac{2 z \zeta P_{0} F_{v}(\zeta+z)}{(\zeta-z)^{3}}\right] .
\end{array}
$$

Since $\operatorname{Re} P_{0}(z)=0$ on $|z|=1$, the corresponding starlike schlicht function $f(z)$ has radial slits. Using the special variation $\delta w= \pm i\left(w-w_{0}\right)$ (where the sign is chosen so $\delta w$ is directed inward) on one side of a slit from $w_{0}$ to $\infty$, and zero everywhere else we have a "hinging" of one of the radial slits at the point $w_{0}$ of the slit. This device has been used by Hummel [7]. $w_{0}$ is chosen far out along the slit so that $Q\left(e^{i \theta}\right)$ is of constant sign on the arc of $|z|=1$ which corresponds to the portion of the radial slit which is varied. $\phi d w$ tends to zero as $w$ goes to infinity on the slit and is constantly negative. We shall presently show that the square bracket of the integrand in (3.9), when $z=r$, has its real part equal to

$$
\frac{r Q(\zeta)}{|\zeta-r|^{6}}, \quad|\zeta|=1
$$

and the quantity (3.10) is real and of constant sign by our choice of $w_{0}$. Since the integrand in (3.9) is of constant sign for $z=r$ and since also $\operatorname{Re} D \geqq 0$ for the extremal function $P_{0}(z)$ when $z=r$ it follows that the square bracket in the integrand in (3.9) must have a negative real part. Since the hinge may be made on either side of the slit, it follows that $Q(\zeta)$ on $|\zeta|=1$ is negative near two consecutive poles $\bar{\varepsilon}_{k}, \bar{\varepsilon}_{k+1}$ of $P_{0}(z)$ on the arc between them. Also since $f^{\prime}(z)=0$ at the tip of each radial slit, $Q(\zeta)$ must vanish somewhere between $\bar{\varepsilon}_{k}, \bar{\varepsilon}_{k+1}$, and in fact vanish an even number of times. So $Q(\zeta)$ has at least $2 m$ zeros but not more than 4 and $2 m \leqq 4, m$ being the number of simple poles of the extremal function $P_{0}(z)$.

There remains then to show that the real part of the square bracket of the integrand in (3.9), when $z=r$, has the value given in (3.10). On $|\zeta|=1$ we have

$$
\operatorname{Re} Q(\zeta)=\alpha_{2}+2 \operatorname{Re}\left[\alpha_{0} \bar{\zeta}^{2}+\alpha_{1} \bar{\zeta}\right], \quad \zeta=e^{i \theta} .
$$


Substituting the values of $\alpha_{2}, \alpha_{0}$ and $\alpha_{1}$ from (2.23), (2.24) and (2.25) we obtain after simplification

$$
\begin{aligned}
\operatorname{Re} Q(\zeta)= & \left(P_{0}^{\prime} F_{u}+P_{0}^{\prime} F_{v}+r P_{0}^{\prime \prime} F_{v}\right)\left(1-r^{2}\right)\left(1-2 r \cos \theta+r^{2}\right)^{2} \\
& -\left(6 r^{2}+6 r^{4}\right)\left\{P_{0}^{\prime} F_{v}+\left(P_{0}^{\prime} F_{v}\right)^{-}\right\} \\
& -\left(3 r+3 r^{3}\right)\left\{P_{0} F_{u}+\left(P_{0} F_{u}\right)^{-}\right\} \\
& +\left(3 r^{3}-3 r\right)\left\{P_{0} F_{v}+\left(P_{0} F_{v}\right)^{-}\right\}-2 \operatorname{Re} C
\end{aligned}
$$

where

$$
\begin{aligned}
C= & r\left(P_{0} F_{u}-P_{0} F_{v}\right) \bar{\zeta}^{2}+r^{3}\left(P_{0} F_{u}+P_{0} F_{v}\right)^{-} \cdot \bar{\zeta}^{2}+2 r^{2} P_{0}^{\prime} F_{v} \bar{\zeta}^{2}+2 r^{4}\left(P_{0}^{\prime} F_{v}\right)^{-} \cdot \bar{\zeta}^{2} \\
& -P_{0} F_{u}\left(3 r^{2}+1\right) \bar{\zeta}-\left(3 r^{2}+r^{4}\right)\left(P_{0} F_{u}\right)^{-} \cdot \bar{\zeta}-\left(6 r^{3}+2 r\right) P_{0}^{\prime} F_{v} \bar{\zeta} \\
& -\left(6 r^{3}+2 r^{5}\right)\left(P_{0}^{\prime} F_{v}\right)^{-} \cdot \bar{\zeta}-\left(1-3 r^{2}\right) P_{0} F_{v} \bar{\zeta}-\left(3 r^{2}-r^{4}\right)\left(P_{0} F_{v}\right)^{-} \cdot \bar{\zeta} .
\end{aligned}
$$

On the other hand the square bracket of the integrand in (3.9), when $z=r$, $\zeta=e^{i \theta}$, has its real part equal to

$$
r \operatorname{Re}\left[\left(r P_{0}^{\prime \prime} F_{v}+P_{0}^{\prime} F_{u}+P_{0}^{\prime} F_{v}\right)\left(\frac{\zeta+r}{\zeta-r}\right)+\frac{2 \zeta F_{u} P_{0}+4 r \zeta F P^{\prime}}{(\zeta-r)^{2}}\right.
$$

$$
=r \operatorname{Re}\left[\begin{array}{l}
\left(r P_{0}^{\prime \prime} F_{v}+P_{0}^{\prime} F_{u}+P_{0}^{\prime} F_{v}\right)\left(1-r^{2}\right) E^{-1}+ \\
E^{-2}\left\{2 \zeta F_{u} P_{0}(\bar{\zeta}-r)^{2}+4 r \zeta(\bar{\zeta}-r)^{2} P_{0}^{\prime} F_{v}\right\}+2\left(\zeta^{2}+r \zeta\right)(\bar{\zeta}-r)^{3} P_{0} F_{v} E^{-3}
\end{array}\right]
$$

where $E=1-2 r \cos \theta+r^{2}=|\zeta-r|^{2}, \quad \zeta=e^{i \theta}$. (3.14) can be rewritten in the form

$$
\begin{aligned}
& r \operatorname{Re}\left[\begin{array}{l}
\left(r P_{0}^{\prime \prime} F_{v}+P_{0}^{\prime} F_{u}+P_{0}^{\prime} F_{v}\right)\left(1-r^{2}\right) E^{-1} \\
+\left\{2\left(\bar{\zeta}-2 r+r^{2} \zeta\right) P_{0} F_{u}+\left(-8 r^{2}+4 r^{3} \zeta+4 r \bar{\zeta}\right) P_{0}^{\prime} F_{v}\right\} E^{-2} \\
+2 P_{0} F_{v}\left\{\left(3 r^{3}-3 r\right)+\left(3 r^{2}-r^{4}\right) \zeta+\left(1-3 r^{2}\right) \bar{\zeta}+r \bar{\zeta}^{2}-r^{3} \zeta^{2}\right\} E^{-3}
\end{array}\right] \\
& =\frac{r}{E^{3}} \operatorname{Re}\left[\begin{array}{l}
+2 P_{0} F_{u}\left\{-3 r-3 r^{3}+\left(3 r^{2}+r^{4}\right) \zeta+\left(1+3 r^{2}\right) \bar{\zeta}-r \bar{\zeta}^{2}-r^{3} \zeta^{2}\right\} \\
+P_{0}^{\prime} F_{v}\left\{-12 r^{2}-12 r^{4}+\left(12 r^{3}+4 r^{5}\right) \zeta+\left(12 r^{3}+4 r\right) \bar{\zeta}-4 r^{4} \zeta^{2}-4 r^{2} \bar{\zeta}^{2}\right\} \\
+2 P_{0} F_{v}\left\{3 r^{3}-3 r+\left(3 r^{2}-r^{4}\right) \zeta+\left(1-3 r^{2}\right) \bar{\zeta}+r \bar{\zeta}^{2}-r^{3} \zeta^{2}\right\}
\end{array}\right] \\
& =\frac{r}{|\zeta-r|^{6}} \operatorname{Re} Q(\zeta), \zeta=e^{i \theta},
\end{aligned}
$$

from (3.12) and (3.13). Here we use the fact that $\operatorname{Re} w=\operatorname{Re} \bar{w}$, and the knowledge that $\left(r P_{0}^{\prime \prime} F_{v}+P_{0}^{\prime} F_{u}+P_{0}^{\prime} F_{v}\right)$ is real. 
This completes the proof of Theorem 1 .

4. Proof of Theorem 2. For the sake of completeness we shall sketch briefly how Theorem 2 follows by the method of proof used in proving Theorem 1 . We shall then give an alternative proof by the method of subordination which is easier and shorter.

If $F(u, v)$ is replaced by the analytic function $F(u), \operatorname{Re} u>0$, we have $F_{v}=0$ in our previous calculations. We have previously noted that in this case the degrees of each of the polynomials in (2.20) and (2.21) are decreased by 2 . For a minimum $\operatorname{Re} F\left(P(r)\right.$ ) we have from Lemma 1 , on setting $F_{v}=0$, the equation

$$
I_{m}\left\{F^{\prime}\left(P_{0}(r)\right) \cdot P_{0}^{\prime}(r)\right\}=0,
$$

where

$$
P_{0}(z)=\frac{\alpha_{0}+\alpha_{1} z+\alpha_{2} z^{2}+\alpha_{3} z^{3}}{\left(F^{\prime}-r^{2} F^{\prime},-2 r\left(F^{\prime}-F^{\prime}\right) z+\left(r^{2} F^{\prime}-F^{\prime}\right) z^{2}\right.}
$$

and $F^{\prime}=F^{\prime}\left\{P_{0}(r)\right\}$. Letting $P_{0}^{\prime}=P_{0}^{\prime}(r)$, we obtain for the coefficients $\alpha_{k}, k$ $=0,1,2,3$ the values

$$
\begin{aligned}
& \alpha_{0}=\left(P_{0}-r P_{0}^{\prime}\right) F^{\prime}+\left(r^{3} \bar{P}_{0}^{\prime}+r^{2} \bar{P}_{0}\right) F^{\prime}, \\
& \alpha_{1}=\left\{\left(1+2 r^{2}\right) P_{0}^{\prime}-2 r P_{0}\right\} F^{\prime}-\left\{\left(2 r^{2}+r^{4}\right) \bar{P}_{0}^{\prime}+2 r \bar{P}_{0}\right\} F^{\prime}, \\
& \alpha_{2}=\left\{r^{2} P_{0}-\left(2 r+r^{3}\right) P_{0}\right\} F^{\prime}+\left\{\bar{P}_{0}+\left(2 r^{3}+r\right) \bar{P}_{0}^{\prime}\right\} F^{\prime}, \\
& \alpha_{3}=-2 i r^{2} I_{m}\left(P_{0}^{\prime} F^{\prime}\right)=0 \quad \text { by }(4.1) .
\end{aligned}
$$

Because of the equation (4.1), $\alpha_{1}=\mathrm{a}$ real number and $\alpha_{2}=\bar{\alpha}_{0}$. Thus (4.2) becomes

$$
P_{0}(z)=\left(\frac{\alpha_{0}}{z}+\alpha_{1}+\bar{\alpha}_{0} z\right) \div\left(\frac{\beta_{0}}{z}+\beta_{1}-\bar{\beta}_{0} z\right)=\frac{Q(z)}{R(z)},
$$

where $\beta_{1}=-2 r\left(F^{\prime}-F^{\prime}\right)=$ a pure imaginary number, and $\beta_{0}=F^{\prime}-r^{2} F^{\prime}$.

Applying (3.8) to $F(u, v)=F(u),(3.9)$ becomes for $z=r$

$$
\begin{aligned}
D & =\oint_{C}\left[r P_{0}^{\prime}(r) F^{\prime}\left(P_{0}\right)\left(\frac{\zeta+r}{\zeta-r}\right)+\frac{2 r \zeta P_{0}(r) F^{\prime}\left(P_{0}\right)}{(\zeta-r)^{2}}\right] \phi d w, \\
\operatorname{Re} D & =\oint_{C} \frac{r \operatorname{Re} Q(\zeta)}{|\zeta-r|^{4}} \phi d w
\end{aligned}
$$

by calculations similar to $\S 3$. Thus we conclude that $2 m \leqq 2$, or $m=1$ in this case. Theorem 2 follows immediately.

However, by the theory of subordination each function $P(z)$ is subordinate to $(1+z)(1-z)^{-1}$ since $\operatorname{Re} P(z)>0$ in $|z|<1$. Hence

$$
P(z)=\frac{1+w(z)}{1-w(z)}
$$


where $w(z)$ is regular and $|w(z)|<1$ in $|z|<1, w(0)=0$. It follows that

$$
F(P)=F\left\{\frac{1+w}{1-w}\right\}, \quad w=w(z) .
$$

Consequently $F(P(z))$ is subordinate to the function $F((1+z) /(1-z))$ in $|z|<1$, and consequently in $|z| \leqq r<1$. (1.22) evidently follows at once, which completes the proof of Theorem 2 .

5. Proofs of Theorems 3 and 4. Let $P(z) \in \mathscr{P}$. We shall apply Theorem 1 to show that

$$
\operatorname{Re}\left\{P(z)-\frac{z P^{\prime}(z)}{P(z)}\right\} \geqq 0 \text { for }|z| \leqq 3^{-1 / 2}=0.577 \cdots,
$$

and that the bound $3^{-1 / 2}$ is sharp. For this special case $F(u, v)=u-v u^{-1}$ in Theorem 1 and the minimum on $|z|=r$ of the left-hand side of inequality (5.1), taken over all members $P \in \mathscr{P}$, occurs for an extremal function of the form

$$
P_{0}(z)=\frac{1+\alpha}{2}\left(\frac{1+z e^{i \theta}}{1-z e^{i \theta}}\right)+\frac{1-\alpha}{2}\left(\frac{1+z e^{-i \theta}}{1-z e^{-i \theta}}\right), z=r e^{i \phi},
$$

where $-1 \leqq \alpha \leqq 1,0 \leqq \theta \leqq 2 \pi, 0 \leqq \phi \leqq 2 \pi$. We must determine the values of the parameters $\alpha, \theta, \phi$ which minimize the left-hand side of (5.1) on $|z|=r$ when $P(z)=P_{0}(z)$. We have

$$
P_{0}(z)-\frac{z P_{0}^{\prime}(z)}{P_{0}(z)}=\frac{2 \alpha i z \sin \theta}{1-2 z \cos \theta+z^{2}}+\frac{1+z^{2}}{1+2 \alpha i z \sin \theta-z^{2}},
$$

$$
\operatorname{Re} \frac{2 \alpha i z \sin \theta}{1-2 z \cos \theta+z^{2}}=\frac{-2 \alpha r\left(1-r^{2}\right) \sin \theta \sin \phi}{\left(1-r^{2}\right)^{2}-4 r(1-r)^{2} \cos \theta \cos \phi+4 r^{2}(\cos \theta-\cos \phi)^{2}}
$$

$$
\operatorname{Re} \frac{1+z^{2}}{1+2 \alpha i z \sin \theta-z^{2}}=\frac{\left(1-r^{2}\right)\left(1-2 \alpha r \sin \theta \sin \phi+r^{2}\right)}{\left\{2 \alpha r \sin \theta-\left(1+r^{2}\right) \sin \phi\right\}^{2}+\left(1-r^{2}\right)^{2} \cos ^{2} \phi}
$$

For $r<1$,

if, and only if,

$$
\mathscr{R}\left\{P_{0}(z)-\frac{z P_{0}^{\prime}(z)}{P_{0}(z)}\right\} \geqq 0
$$

$\left(1-2 \alpha r \sin \theta \sin \phi+r^{2}\right)\left[\left(1-r^{2}\right)^{2}-4 r(1-r)^{2} \cos \theta \cos \phi+4 r^{2}(\cos \theta-\cos \phi)^{2}\right]$

$$
-2 \alpha r \sin \theta \sin \phi\left[\left\{2 \alpha r \sin \theta-\left(1+r^{2}\right) \sin \phi\right\}^{2}+\left(1-r^{2}\right)^{2} \cos ^{2} \phi\right] \geqq 0 .
$$

Since $\left(1-2 \alpha r \sin \theta \sin \phi+r^{2}\right) \geqq(1-r)^{2}>0$ and $\left(1-r^{2}\right)^{2}-4 r(1-r)^{2} \cos \theta \cos \phi$ $\geqq(1-r)^{4}>0$, we need only consider the inequality (5.7) in the case $\alpha \sin \theta \sin \phi$ $>0$. Thus $\alpha \sin \theta$ and $\sin \phi$ have the same sign. Since

$$
\left[\left(1-r^{2}\right)^{2}-4 r(1-r)^{2} \cos \theta \cos \phi+4 r^{2}(\cos \theta-\cos \phi)^{2}\right]
$$


is smallest when $\cos \theta$ and $\cos \phi$ have the same sign, it is evident that we need consider the inequality (5.7) only for the case

$$
0<\alpha \leqq 1, \quad 0<\theta \leqq \pi / 2, \quad 0<\phi \leqq \pi / 2 .
$$

We make the substitution

$$
2 \sigma=r+r^{-1} \geqq 2, \quad r=\sigma-\left(\sigma^{2}-1\right)^{1 / 2}
$$

in (5.7) which becomes

$$
F=F(\sigma, \alpha, \theta, \phi) \geqq 0
$$

where

$$
\begin{aligned}
F= & \sigma^{3}-2(\cos \theta \cos \phi+\alpha \sin \theta \sin \phi) \sigma^{2} \\
& +\left[(\cos \theta \cos \phi+\alpha \sin \theta \sin \phi)^{2}-\left(1-\alpha^{2}\right) \sin ^{2} \theta \sin ^{2} \phi\right] \sigma \\
& +\left(\alpha-\alpha^{3}\right) \sin ^{3} \theta \sin \phi \\
= & \sigma[\sigma-(\cos \theta \cos \phi+\alpha \sin \theta \sin \phi)]^{2}-\left(1-\alpha^{2}\right) \sin ^{2} \theta \sin ^{2} \phi \cdot \sigma \\
& +\left(\alpha-\alpha^{3}\right) \sin ^{3} \theta \sin \phi .
\end{aligned}
$$

$F=0$ has at least one real root $\sigma$. Let $\sigma=\sigma(\alpha, \theta, \phi)$ be the largest real zero of $F$ for fixed $\alpha, \theta, \phi$. Let

$$
\sigma_{0}=\max _{\alpha, \theta, \phi} \sigma(\alpha, \theta, \phi)=\sigma\left(\alpha_{0}, \theta_{0}, \phi_{0}\right)
$$

If $\alpha=1, F$ has zeros $\sigma=0, \sigma=\cos (\theta-\phi)$ (a double root). Since $\sigma_{0}$ is known to be at least 1 we can therefore rule out the possibility $\alpha=1$. If $\phi=\pi / 2, F$ has zeros $\sigma=\alpha \sin \theta$,

$$
\sigma=\alpha / 2 \pm \frac{1}{2}\left(4-3 \alpha^{2}\right)^{1 / 2} \sin \theta
$$

whose maximum, occurring for $\theta=\pi / 2, \alpha=3^{-1 / 2}$ is $\sigma=2(3)^{-1 / 2}>1$. If there exists a $\sigma_{0}>2(3)^{-1 / 2}$ we can assume

$$
0<\alpha<1, \quad 0<\theta \leqq \pi / 2, \quad 0<\phi<\pi / 2 .
$$

From (5.10) we obtain

$$
\sigma=\cos \theta \cos \phi+\sin \theta \sin \phi\left[\alpha+\left\{\left(1-\alpha^{2}\right)\left(1-\frac{\alpha \sin \theta}{\sigma \sin \phi}\right)\right\}^{1 / 2}\right] .
$$

For a maximum $\sigma$ we must have from (5.11)

$$
\frac{\partial \sigma}{\partial \theta}=0
$$

Because of (5.12) when we differentiate $\sigma$ in (5.11) we may treat the $\sigma$ on the right-hand side of (5.11) as if this $\sigma$ were a constant. We obtain 
(5.13) $\sin \theta \cos \phi=\cos \theta \sin \phi\left[\alpha+\frac{\left(1-\alpha^{2}\right)^{1 / 2}}{2}\left(2-\frac{3 \alpha}{\sigma} \frac{\sin \theta}{\sin \phi}\right)\left(1-\frac{\alpha}{\sigma} \frac{\sin \theta}{\sin \phi}\right)^{1 / 2}\right]$.

Similarly $\partial \sigma / \partial \alpha=0$ gives us

$$
\frac{\sin ^{2} \phi}{\sin ^{2} \theta}-\frac{2 \alpha}{\sigma} \frac{\sin \phi}{\sin \theta}-\frac{\left(1-3 \alpha^{2}\right)^{2}}{4 \sigma^{2}\left(1-2 \alpha^{2}\right)}=0 .
$$

Since $\sigma \sin \phi / \sin \theta>0,(5.14)$ gives

$$
\frac{\sigma \sin \phi}{\sin \theta}=\alpha+\frac{1}{2}\left(1-\alpha^{2}\right)\left(1-2 \alpha^{2}\right)^{-1 / 2} .
$$

Again, $\partial \sigma / \partial \phi=0$ gives

$$
\cos \theta \sin \phi=\sin \theta \cos \phi\left[\alpha+\frac{1}{2}\left(1-\alpha^{2}\right)^{1 / 2}\left(2-\frac{\alpha}{\sigma} \frac{\sin \theta}{\sin \phi}\right)\left(1-\frac{\alpha}{\sigma} \frac{\sin \theta}{\sin \phi}\right)^{-1 / 2}\right]
$$

By virtue of the equation (5.15), the equations (5.13) and (5.16) may be rewritten as the following equations (5.17) and (5.18).

$$
\begin{aligned}
& \sin \theta \cos \phi=\sin \phi \cos \theta\left[\alpha+\left(1-2 \alpha^{2}\right)^{1 / 2}\right]^{-1} \\
& \sin \phi \cos \theta=\sin \theta \cos \phi \frac{1+2 \alpha\left(1-2 \alpha^{2}\right)^{1 / 2}}{\alpha+\left(1-2 \alpha^{2}\right)^{1 / 2}}
\end{aligned}
$$

If $\theta=\pi / 2$ then (5.17) (or (5.18)) gives $\phi=\pi / 2$ which leads to $\sigma=2(3)^{-1 / 2}$ as we have seen before. If $0<\theta<\pi / 2,0<\phi<\pi / 2$, (5.17) and (5.18) are compatible only if

$$
\left[\alpha+\left(1-2 \alpha^{2}\right)^{1 / 2}\right]^{-1} \cdot\left[1+2 \alpha\left(1-2 \alpha^{2}\right)^{1 / 2}\right]\left[\alpha+\left(1-2 \alpha^{2}\right)^{1 / 2}\right]^{-1}=1 .
$$

But (5.19) is satisfied only if $\alpha=0$. Thus

$$
\frac{\partial \sigma}{\partial \theta}, \frac{\partial \sigma}{\partial \alpha}, \frac{\partial \sigma}{\partial \phi}
$$

cannot all vanish if $0<\alpha<1,0<\theta<\pi / 2,0<\phi<\pi / 2$. If $\partial \sigma / \partial \phi \neq 0$ then $\max \sigma$ occurs at $\phi=\pi / 2$ in which case $\sigma_{0}=2(3)^{-1 / 2}$. If $\partial \sigma / \partial \alpha \neq 0$ the $\max \sigma$ would have to occur at $\alpha=0$ or 1 , but this case has been ruled out. If $\partial \sigma / \partial \theta \neq 0$, then $\theta=\pi / 2$. In this case if the $\max \sigma$ does not occur when $\phi=\pi / 2$ then $\partial \sigma / \partial \phi$ must vanish. Also $\partial \sigma / \partial \alpha$ must vanish (otherwise $\alpha=0$ or 1). Thus (5.15) and (5.18) hold when $\theta=\pi / 2$. (5.18) then forces $\phi=\pi / 2$.

It is seen that if $\alpha \neq 0,(5.15),(5.17)$ and (5.18) are compatible only when $\phi=\theta=\pi / 2$. Hence $\sigma_{0}=2(3)^{-1 / 2}$. Then (5.15) gives $\alpha=3^{-1 / 2}$.

Since $F$ has no zeros $\sigma$ larger than $2(3)^{-1 / 2}$ and since $F$ is a cubic polynomial with leading coefficient positive, it follows that $F \geqq 0$ for $\sigma \geqq 2(3)^{-1 / 2}$ with equality occurring only if $\theta=\phi=\pi / 2, \alpha=3^{-1 / 2}$. For $\sigma=2(3)^{-1 / 2}$ we have $r=3^{-1 / 2}$. 
The extremal function (5.2) becomes

$$
P_{0}(z)=\frac{1+3^{-1 / 2}}{2}\left(\frac{1+i z}{1-i z}\right)+\frac{1-3^{-1 / 2}}{2}\left(\frac{1-i z}{1+i z}\right),
$$

and equality holds in (5.1) for $P(z)=P_{0}(z), z=3^{-1 / 2} \cdot i$. We may obviously replace $P_{0}(z)$ by $P(-i \varepsilon z),|\varepsilon|=1$, so that this completes the proof of Theorem 3 .

As we pointed out in the introduction Theorem 4 follows from Theorem 3 because of the equation (1.6). The extremal function $F_{0}(z)$ of $(1.26)$ is obtained from the solution (subject to normalization) of the equation

$$
\frac{z F_{0}^{\prime}(z)}{F_{0}(z)}=-P_{0}(z)
$$

where $P_{0}(z)$ is defined by (1.24). $F_{0}(z)$ maps the unit circle onto a slit domain, the complex plane minus two rectilinear slits each of length

$$
2\left[(1+\alpha)^{1+\alpha} \cdot(1-\alpha)^{1-\alpha}\right]^{1 / 2}, \quad \alpha=3^{-1 / 2},
$$

and subtending at the origin an angle $\pi\left(1-3^{-1 / 2}\right)$.

6. Proof of Theorem 5. In the introduction we made use of the inequality (1.15) or

$$
\operatorname{Re}\left[\frac{i \sin \mu z P^{\prime}(z)}{\cos \mu+i \sin \mu P(z)}\right] \geqq \frac{-2 r}{1-r^{2}},|z|=r<1 .
$$

By Theorem 1 the extremal function, associated with this special case involving $F(u, v)$ of (1.16), must be of the form (1.21) from which (6.1) may be deduced. However, the following alternative method of proof is more instructive and yields at the same time the stronger inequality

$$
\left|\frac{\sin \mu z P^{\prime}(z)}{P \sin \mu-i \cos \mu}\right| \leqq \frac{\left|z P^{\prime}(z)\right|}{\operatorname{Re} P(z)} \leqq \frac{2 r}{1-r^{2}}, \quad r<1 .
$$

Define the analytic function $\phi(\zeta)$ by the equation

$$
\begin{aligned}
\phi(\zeta) & =\frac{P((\zeta+z) /(1+\bar{z} \zeta))-i I_{m} P(z)}{\operatorname{Re} P(z)}, \quad \phi(0)=1, \quad|\zeta|<1 . \\
\phi^{\prime}(0) & =\frac{P^{\prime}(z)\left(1-|z|^{2}\right)}{\operatorname{Re} P(z)} .
\end{aligned}
$$

Since $\operatorname{Re} \phi(\zeta)>0,|\zeta|<1$, we have $\left|\phi^{\prime}(0)\right| \leqq 2$.

$$
\frac{\left|z P^{\prime}(z)\right|}{\operatorname{Re} P(z)}=\frac{\left|z \phi^{\prime}(0)\right|}{1-|z|^{2}} \leqq \frac{2|z|}{1-|z|^{2}}=\frac{2 r}{1-r^{2}} .
$$

(6.2) follows from (6.5) and is sharp for $\mu=\pi / 2$ and 


$$
P(z)=(1+\varepsilon z)(1-\varepsilon z)^{-1}, \quad|\varepsilon|=1 .
$$

Theorem 5 results from the inequality (1.14) which follows from (6.2).

\section{REFERENCES}

1. S. Gelfer, Sur les bornes de l'étoilement et de la convexité des fonctions p-valentes, Mat. Sb. (N. S.) 58 (16) (1945), 81-86.

2. G. M. Golusin, Über einige Abschatzungen von Funktionen, welche den Kreis konform und schlicht abbilden, Mat. Sb. (N. S.) 36 (1929), 152-172.

3. - Z Zur theorie der schlichten konformen Abbildungen, Mat. Sb. (N. S.) 42 (1935), 169-190.

4. hängender Bereiche, Mat. Sb. (N. S.) 2(44) (1937), 37-64.

5. —_ Zur Theorie der schlichten Functionen, Mat. Sb. (N. S.) 12 (54) (1943), 48-55.

6. - Geometrische Funktionentheorie, Deutscher Verlag, Berlin, 1957.

7. J. A. Hummel, $A$ variational method for starlike functions, Proc. Amer. Math. Soc. 9 (1958), 82-87.

8. - Extremal problems in the class of starlike functions, Proc. Amer. Math. Soc. 11 (1960), 741-749.

9. G. Julia, Sur une équation aux dérivées fonctionnelles liée à la représentation conforme, Ann. Sci. École Norm. Sup. 39 (1922), 1-28.

10. R. Nevanlinna, Über die schlichten Abbildungen des Einheitskreises, Oversikt av Finska Vet. Soc. Förh. 62 (1919-1920), 1-14.

11. M. S. Robertson, Analytic functions starlike in one direction, Amer. J. Math. 58 (1936), 465-472.

12. - Variational methods for functions with positive real part, Trans. Amer. Math. Soc. 101 (1962), 82-93.

Rutgers, The State University,

NeW Brunswick, NeW Jersey 